\title{
MINIMAL CLINICALLY IMPORTANT DIFFERENCES IN HEALTH-RELATED QUALITY OF LIFE AFTER ISCHEMIC STROKE
}

\author{
Mihael Tsalta-Mladenov \\ Department of Neurology and Neuroscience, Faculty of Medicine, \\ Medical University of Varna \\ Second Clinic of Neurology with ICU and Stroke Unit, \\ St. Marina University Hospital, Varna
}

\begin{abstract}
INTRODUCTION: Health-related quality of life (HR-QoL) is an essential index of outcome after acute ischemic stroke (AIS). The minimal clinically important differences (MCIDs) represent the smallest change detected by a specific HR-QoL measuring tool beyond the standard measurement error.

AIM: We aimed to establish the MCID in the HR-QoL after AIS during the first three months post-stroke.

MATERIALS AND METHODS: A total of 143 participants were assessed using the Stroke Impact Scale 3.0 (SIS 3.0) to measure HR-QoL at discharge, the first and the third month after AIS. Aggregated scores between 0-100 for all domains were used. MCID was defined as positive changes (PCs)(+15 points), negative changes (NCs)(-15 points), and no significant difference (NSDs)(-14 to +14). General linear modeling was used to determine time changes, and the differences were considered significant at a p-value $<0.05$.
\end{abstract}

RESULTS: The most affected SIS 3.0 domains on the third month were Mobility - 56.74, Hand function 58.92, and Strength - 59.62. The majority of the patients had NSDs. The proportion of those with NCs was significantly lower than those of PCs. The domains with most PCs were Stroke recovery - 34.97\%, Strength $33.57 \%$, and Mobility - 31.47\%, while Memory and thinking had the least $-13.29 \%$. The most NCs were found in the domains Participation - 4.90\%, Emotion - 4.20\%, and Hand function -3.50\%. The emotion domain manifested with the greatest variety ranging from $25.87 \%$ PCs and $4.20 \%$ NCs.

CONCLUSION: The most affected HR-QoL aspects were from the physical dimension, with an additionally high level of engagement of the emotional sphere. Stroke survivors require a holistic approach during the recovery period, including physical and speech rehabilitation and timely social and mental support.

Keywords: health-related quality of life, quality of life, ischemic stroke, stroke, stroke impact scale, minimal clinically important differences, Bulgaria

Address for correspondence:

Mihael Tsalta-Mladenov

Faculty of Medicine

Medical University of Varna

55 Marin Drinov St

9002 Varna

e-mail:mihaeltsalta@gmail.com

Received: August 7, 2020

Accepted: September 15, 2020

\section{INTRODUCTION}

Stroke is one of the leading causes of death and chronic disability worldwide (1). In the last decades, the global incidence of ischemic stroke has increased by $20 \%$ in the middle- and low-income countries (2). In Bulgaria, stroke is a constant leading cause of mortality and disability as the country ranks first in the EU in terms of the stroke mortality rates (3). 
Ischemic stroke is a neurological disease with an immense impact on the stroke survivors' lives. It causes lifetime disabilities with the consequences of functional difficulties and severe activity limitations (4). The assessment of recovery from an ischemic stroke should include different areas (domains) of their quality of life (QoL), which are affected by the disease.

There is rising interest in the health-related quality of life (HR-QoL) after acute ischemic stroke (5). The information collected from the stroke survivors' perspective on the consequences of stroke is critical for evaluating the recovery process. Many disease-specific tools are developed to provide information about the difficulties patients who survived an acute ischemic stroke may experience (6).

Despite the need to determine the overall level of QoL, other properties such as the minimal clinically important difference (MCID) are also of great importance. MCID is the smallest change detected by a certain HR-QoL measuring tool beyond the standard measurement error (7). This parameter facilitates the interpretation of treatment outcomes and allows individual groups' delimitation with minimal positive or negative change over time.

There is a lack of information about the consequences of acute ischemic stroke in Bulgaria. This is the first study to establish HR-QoL after acute ischemic stroke and determine MCIDs during the first three months post-stroke.

\section{MATERIALS AND METHODS Study Design and Participants}

We conducted a prospective, hospital-based study undertaken from July 2019 to June 2020 in the Second Clinic of Neurology with ICU and Stroke Unit at the "St. Marina" University Hospital, Varna. This center is the largest one providing differentiated treatment of acute stroke patients, including thrombolysis and mechanical thrombectomy, in Eastern Bulgaria.

Consecutive patients with first-ever and recurrent acute ischemic stroke (AIS) hospitalized in our clinic were included in the present study. At baseline we recruited a total of 150 participants - 50 patients with thrombolytic therapy and 100 - with non-thrombolytic treatment. We used the American Heart Association/American Stroke Association (AHA/ASA) definition of stroke (8). Ethical permis- sion was obtained from the ethics committee of the Medical University of Varna. All participants provided signed informed consent.

We accepted the following inclusion criteria: age of 18 years or more; acute onset of neurological symptoms lasting more than 24 hours; conducted neuroimaging examination of the head - computed tomography (CT) or magnetic resonance imaging (MRI); and signed informed consent.

Exclusion criteria were: age under 18 years; lack of neurological symptoms or the presence of such was less than 24 hours; refusal to sign an informed consent.

Patients with a transient ischemic attack (TIA) were not included in the present study.

\section{Clinical Evaluation and Measures}

A neurologist similarly interviewed all participants to get accurate sociodemographic data, information for comorbidities, and stroke risk factors. Medical records were checked, and relatives and caregivers were also interviewed. All participants underwent a detailed medical examination with thorough neurological status, blood tests, and neuroimaging $\mathrm{CT}$ on admission.

Stroke severity was measured on admission and at discharge from the clinic using the National Institutes of Health Stroke Scale (NIHSS) (9). Disability and functional independence of patients were measured using the modified Rankin Scale (mRS) (10). Stroke survivors were categorized as functionally independent (mRS 0-2 points) and functionally dependent (mRS 3-5 points) in their daily activities.

The Stroke Impact Scale version 3.0 (SIS 3.0) (11) was applied to evaluate HR-QoL on discharge and at two follow-ups - at the first and the third month post-stroke. SIS 3.0 is a comprehensive specific measure for QoL in post-stroke patients. This scale has been proven suitable for a post-stroke patient followup, as it has high reliability, sensitivity, and reproducibility for each domain (6). It consists of 59 items in 8 domains - Strength, Hand function, Activities of daily living (ADL), Mobility, Communication, Emotion, Memory and thinking, and Participation (12). The first four of the domains are combined in a composite physical domain score. Each item is being rated on a five-point Likert scale, where 1 stands for inability to complete the item, and 5 is equal to no dif- 
ficulties at all. There is a total score for each domain and an overall score for the whole questionnaire. Patients self-report the recovery from stroke using a visual analogue scale (VAS) ranging from 0 - no recovery, to 100 denoting a full recovery. In this study, we used the domain-specific and total SIS 3.0 score for the measure of HR-QoL.

There is no defined range of the change to determine MCID for SIS 3.0. Nevertheless, numerous studies have characterized the MCID range as $10 \%$ of the total range of scales. Other researchers set MCID in the range between $10 \%$ and $15 \%$ based on clinical experience (4). Our study accepted MCIDs as a change of $15 \%$ of the total range scale. Subsequently, we defined positive changes (PCs) (+15 points), negative changes (NCs) ( -15 points), and no significant differences (NSDs) (between -14 to +14 ).

\section{Statistical Analysis}

The data analysis was carried out using gold standard methods widely reported in the HR-QoL literature (12). We analyzed the HR-QoL data obtained at all three time points. The items were recalibrated so that a high score always indicates better HR-QoL. The domain results were later rescaled by the following equation - Domain score $=[$ (Mean item score - 1) / 5-1] x 100. The calculated final scores have a standard range from 0 to 100 , where a score of 100 denotes the best health (4). The overall HR-QoL score was generated by finding the arithmetic mean of all the domain scores.

The statistical analysis included descriptive and inferential statistics. A comparison of the participants' responses was made using ANOVA and correlation statistics. General linear modeling was used to measure the changes in all SIS 3.0 domains over time. Wherever possible, confidence intervals (CIs) were calculated. Statistical significance was defined as a p-value (alpha) of 0.05 or less.

Data were analyzed using the software programs SPSS, version 23.00 (IBM Statistics, USA) and GraphPad Prism, version 5.00 (GraphPad Software, San Diego California, USA).

\section{RESULTS}

\section{Baseline Characteristics}

At baseline, there were 150 participants. During the three-month follow-up period, in 7 of the pa- tients were registered fatal outcomes, representing a lethal rate of $4.67 \%$. The final sample comprised 143 participants.

The mean age of the ischemic stroke survivors was $68.54 \pm 11.44$ years, comprising 88 males and 55 females. One-third of the participants we treated with thrombolysis. Predominantly the patients were with a first-ever stroke - 111 cases, and less than a quarter were with a recurrent stroke -32 of them. Most of the patients had a moderate and minor stroke, as the mean NIHSS score on admission was $7.67 \pm 4.51$ and $4.59 \pm 3.59$ on discharge. The mean mRS score at discharge was $2.52 \pm 1.41$ as $44.8 \%$ of the patients were functionally dependent in their daily activities. De-

Table 1. Patient characteristics at baseline

\begin{tabular}{|c|c|c|c|}
\hline \multicolumn{4}{|c|}{ Baseline Characteristics } \\
\hline \multicolumn{2}{|c|}{ Characteristics } & \multicolumn{2}{|c|}{$\begin{array}{c}\text { Cases } \\
(n=143)\end{array}$} \\
\hline & & $\mathbf{N}$ & $\%$ \\
\hline \multirow[t]{2}{*}{ Age } & Mean (years) & \multicolumn{2}{|c|}{$68.54 \pm 11.44$} \\
\hline & $>65$ years & 90 & 62.9 \\
\hline \multirow{2}{*}{ Sex } & Male & 88 & 61.5 \\
\hline & Female & 55 & 38.5 \\
\hline \multirow{2}{*}{ Ischemic stroke } & First-ever stroke & 111 & 77.6 \\
\hline & Recurrent stroke & 32 & 22.4 \\
\hline \multirow{4}{*}{ Localization } & Anterior circulation & 73 & 51.1 \\
\hline & Left hemisphere & 44 & 30.8 \\
\hline & Right hemisphere & 29 & 20.3 \\
\hline & Posterior circulation & 72 & 48.9 \\
\hline \multirow[t]{4}{*}{$\begin{array}{l}\text { Stroke severity } \\
\text { on admission }\end{array}$} & Mean (NIHSS) & \multicolumn{2}{|c|}{$7.67 \pm 4.51$} \\
\hline & Minor stroke (1-4) & 39 & 27.3 \\
\hline & $\begin{array}{l}\text { Moderate stroke } \\
(5-15)\end{array}$ & 90 & 62.9 \\
\hline & $\begin{array}{l}\text { Moderate to severe } \\
\text { stroke }(16-20)\end{array}$ & 14 & 9.8 \\
\hline $\begin{array}{l}\text { Stroke severity } \\
\text { on discharge }\end{array}$ & Mean (NIHSS) & \multicolumn{2}{|c|}{$4.59 \pm 3.59$} \\
\hline \multirow{3}{*}{$\begin{array}{l}\text { Level of } \\
\text { dependence } \\
\text { on discharge }\end{array}$} & Mean (mRS) & \multicolumn{2}{|c|}{$2.52 \pm 1.41$} \\
\hline & $\begin{array}{l}\text { Functional } \\
\text { independence }\end{array}$ & 79 & 55.2 \\
\hline & $\begin{array}{l}\text { Functional } \\
\text { dependence }\end{array}$ & 64 & 44.8 \\
\hline \multirow[b]{2}{*}{ Acute treatment } & Thrombolysis & 48 & 33.6 \\
\hline & $\begin{array}{l}\text { Non-thrombolytic } \\
\text { therapy }\end{array}$ & 95 & 66.4 \\
\hline
\end{tabular}


Table 2. Dynamics in HR-QoL in the different periods of the study

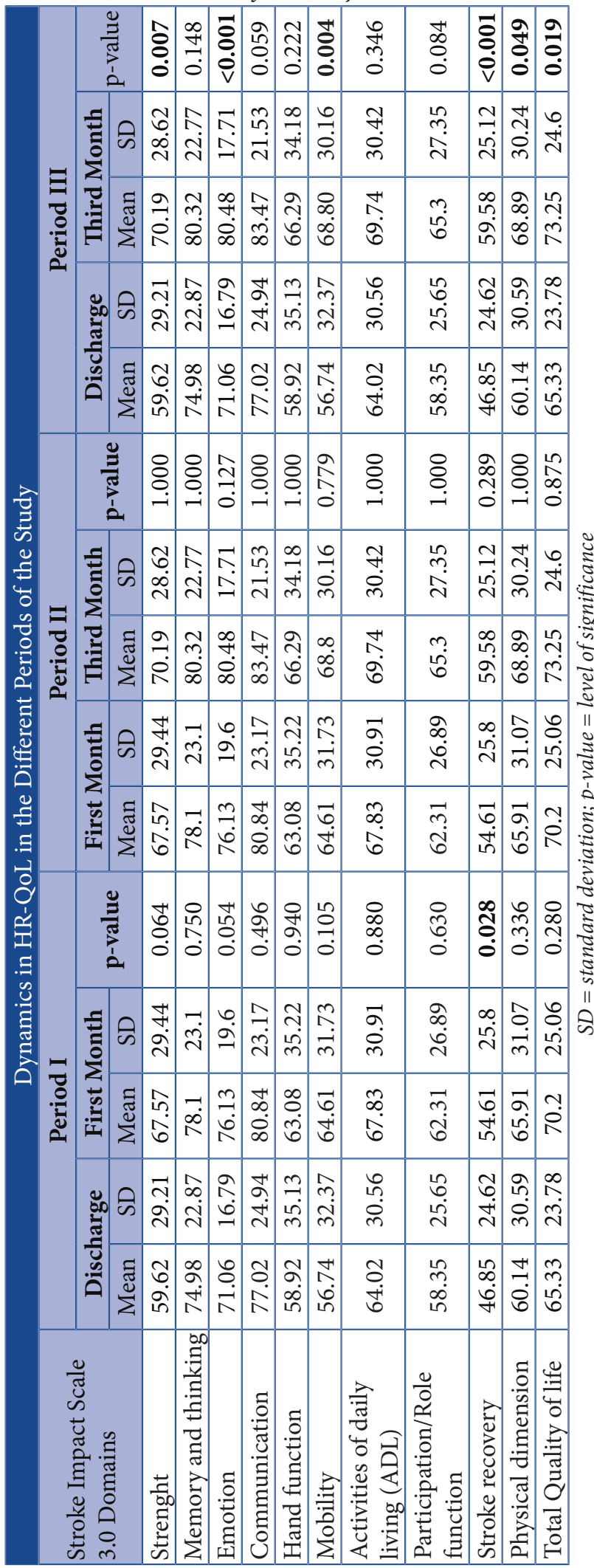

tailed baseline characteristics of the participants are presented in Table 1.

\section{HR-QoL After Ischemic Stroke}

All participants reported a significantly reduced HR-QoL at discharge as the mean total QoL was $65.33 \pm 23.78$. The lowest results were found in the following domains: Mobility - 56.74, Hand function - 58.92, and Strength - 59.62. The mean self-assessed stroke recovery was 45.27 , which indicated the severe post-stroke consequences for the survivors.

During the time between discharge and the first month (Period I), all the domains of SIS 3.0 showed changes, however, the only statistically significant change was in the stroke recovery domain ( $\mathrm{p}=0.028)$.

In the second period the trend of improvement in all domains continued, but to a lesser extent. The general linear modeling showed an advance in all studied fields by the SIS 3.0, without reaching a statistical significance. The Emotion domain presented with the most remarkable change but yet without a significant rate $(\mathrm{p}=0.127)$.

The most significant HR-QoL changes were noted between the discharge and the third month after AIS. Improvement in all aspects of life studied by the SIS 3.0 was observed. The most notable improvement was found in the Emotion domain and the selfreported stroke recovery $(\mathrm{p}<0.001)$. Consequently, Mobility $(\mathrm{p}=0.004)$ and Strength $(\mathrm{p}=0.007)$ were the domains with the most considerable improvement. Finally, the total physical dimension $(p=0.049)$ and the total QoL $(\mathrm{p}=0.019)$ presented significant improvement.

Detailed information regarding the dynamics of HR-QoL in the three periods are presented in Table 2.

Minimal Clinically Important Differences (MCIDs)

The MCIDs analysis shows some main trends within the three studied periods. Throughout the study period, the proportion of patients with NSDs was highest, and the percentage of patients with NCs was consistently low.

Within the first period, the most considerable portion of patients with PCs was found in the Strength and Stroke recovery domains - $18.88 \%$ each, followed by Hand function - 17.48\%, and Participation $-14.69 \%$. For the same period, the lowest PCs were denoted in the Memory and thinking and the 
Mihael Tsalta-Mladenov

whole physical dimension- $6.29 \%$. Only $2.8 \%$ of patients had PCs in the overall QoL. The NCs were most pronounced in the Emotion domain - 5.59\%, followed by Hand function and Participation with $2.10 \%$, respectively.

During the second period, significantly smaller differences in mean changes were reported in all SIS 3.0 domain. The proportion of participants with NSD during this period significantly dominated. The highest PCs were reported in Stroke recovery $-13.29 \%$, followed by Hand function $-8.39 \%$ and Emotion and Mobility respectively by $6.99 \%$. A minor share of the participants was with NCs, as this was more pronounced for the domains Memory and thinking, Emotion, and Participation by $1.40 \%$, respectively.

The third period includes aggregated data for MCIDs from discharge until the end of the third month after the stroke. As expected, due to the longer duration, the most significant changes are reported here. PCs covered up to one-third of the studied population concerning the domains Stroke recovery - $34.97 \%$, Strength - $33.57 \%$, and Mobility $31.47 \%$. The domain of Memory and thinking had the lowest level of PCs $-13.29 \%$. A total of $18.18 \%$ of patients reported a significant improvement in their overall QoL after ischemic stroke. The proportion of patients with NCs was significantly lower than that of PCs. The most pronounced NCs were found in the domains Participation - 4.90\%, Emotion - 4.20\%, and Hand function $-3.50 \%$.

Detailed information on the distribution of MCIDs in the different periods is presented in Table 3.

\section{DISCUSSION}

The present study examined the HR-QoL in adult ischemic stroke survivors and evaluated the MCIDs distribution.

Our results emphasize the severe impairment in the Health-related Quality of life (HR-QoL) assessed with the SIS 3.0. All subscales of this strokespecific tool showed a variable degree of affection in the different domains. The most affected fields at the end of the study period were Hand function

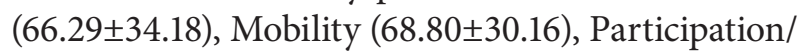
Role function $(65.3 \pm 27.35)$, and the whole physical dimension (68.89 \pm 30.24$)$. Similar results were found
Table 3. Minimal clinically important difference (MCID) in the different periods

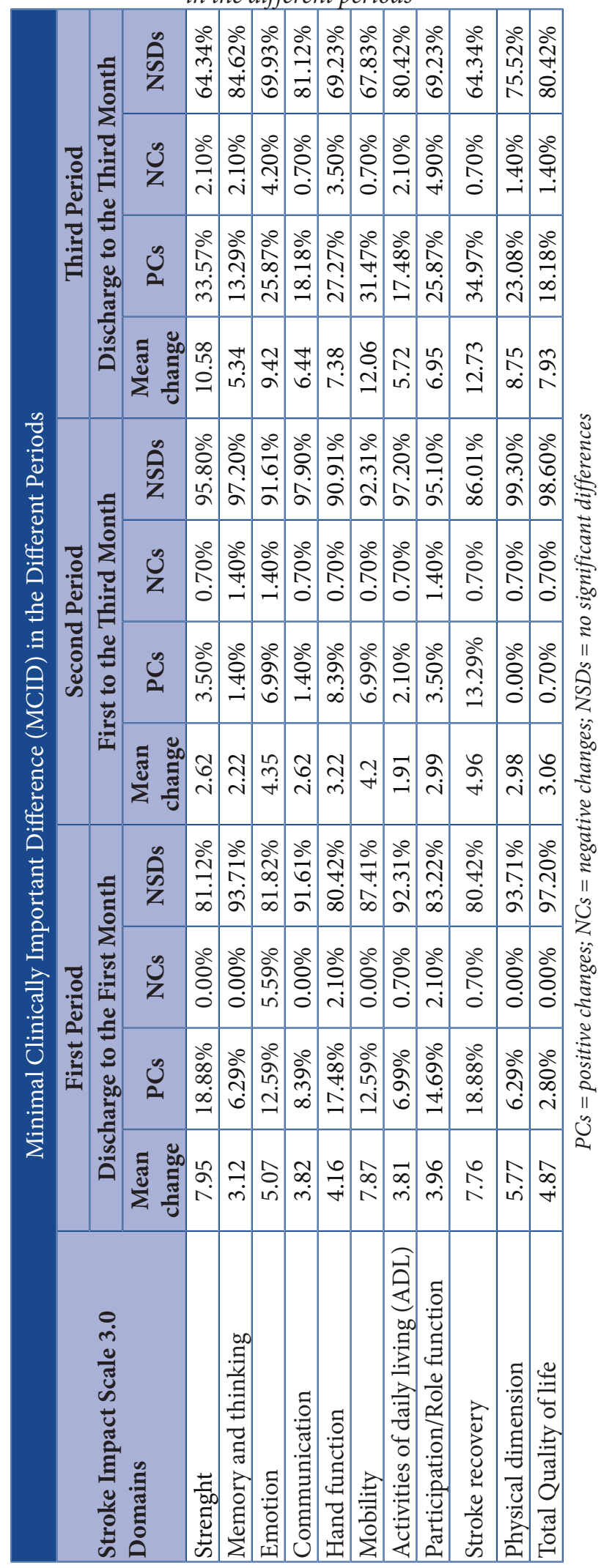


in other HR-QoL studies $(13,14)$. Patients with more severe alterations in the physical dimension were found to have a much worse total QoL in the third month. Perhaps these findings can be explained by the fact that physical disability is the primary concern of most stroke patients because of its direct effect on their ADL and hence, HR-QoL. On the other hand, it has been proven that stroke causes a sufficient decrease in HR-QoL, even in those who do not have severe physical symptoms and disability (12).

Tracking the changes in HR-QoL, we found that it remains significantly decreased till the third month post-stroke. The mean values dynamics were in a broader range during the first period, proving the faster recovery in the early period than the late periods (15). There was much quicker functional improvement in the first month due to the recovery of neurotransmission and neuromodulation in the preserved brain tissue near the ischemic zone (16). After the first month, the recovery tended to slow down and reach a plateau between the third and the sixth month post-stroke (12). The domain with the most significant recovery rates was Emotion $(\mathrm{p}<0.001)$. It was followed by Mobility $(\mathrm{p}=0.004)$, Strength $(\mathrm{p}=0.007)$, the overall physical dimension $(\mathrm{p}=0.049)$, and the total QoL (0.019). These findings are consistent with previous studies reporting the most intensive dynamics in the emotional and physical domains (17). A plausible explanation for these results could be the post-stroke depression and the effect of post-stroke rehabilitation.

MCIDs represent the proportion of the patients who had positive, negative, or no changes in their condition during follow-up according to the SIS 3.0 scale. Determining MCID is crucial for judging whether there is a real change in HR-QoL and its magnitude (18). There is no defined range of the change score in MCID. Nevertheless, previous studies determined MCID as $10 \%$ of the scales' entire range for hemiparetic patients (19) and $10-15 \%$ for the general stroke population (4). We used a broader range of $15 \%$ difference in the total domain score as an MCID measure.

Our study proves that most patients have no significant difference in their HR-QoL and the individual domains of SIS. Nevertheless, there is a preva- lence of patients with PCs over those with NCs during the first three months post-stroke.

During the first period, we found more patients with PCs in the physical domains - Strength, Hand function, Mobility, and Participation. These results are consistent with previous reports (20). The explanation of these findings is the early initiation of physical rehabilitation and continuation of its implementation after discharge. We found pronounced changes in the Emotion domain, as the most considerable portion of NCs were in this domain during this first period. While $12.59 \%$ of the patients were with PCs, there were $5.59 \%$ of them with NCs. The larger share of NCs can be explained with post-stroke depression, a common finding with a prevalence of $29 \%$ anytime after stroke (21). Only minor dynamics were observed in the second period, as the changes in the PC and NC groups were less than $10 \%$ of all the participants. At the end of the study period, we again found the most pronounced physical dimension changes. We noted that out of all patients with PCs, the smallest was the share of those with improvement in Memory and thinking, Communication, and ADL. These results highlight the persistent impairment of cognitive function and memory dysfunction after stroke (22). Therefore, for patients who have suffered a stroke, it is essential to conduct not only physical rehabilitation but also speech rehabilitation and timely social and mental support.

\section{Limitations:}

We acknowledge the following limitations in our study. First, our cohort represents patients predominantly with minor and moderate stroke. Other aspects related to stroke outcomes like psychoemotional disorders, family, and social support can also influence the HR-QoL and were not analyzed in the present study. We used a constant limit of $15 \%$ to determine MCID, while this value may vary for individual domains on the SIS 3.0 scale. Consequently, the results likely underestimate the full impact of stroke on the wellbeing of stroke survivors.

\section{CONCLUSION}

Despite the trend to improve over time, the HRQoL after stroke remains severely impaired up to the third month post-stroke. The distribution of patients with MCIDs proved that the physical aspects of HR- 
QoL are the most affected, with an additional high level of engagement of the emotional sphere.

Stroke survivors require a holistic approach during the recovery period after the acute accident. They need to conduct both physical and speech rehabilitation and to receive timely social and mental support.

\section{REFERENCES}

1. Donkor ES. Stroke in the 21(st) century: A snapshot of the burden, epidemiology, and quality of life. Stroke Res Treat. 2018;2018:3238165. doi: $10.1155 / 2018 / 3238165$.

2. Lackland DT, Roccella EJ, Deutsch AF, Fornage M, George MG, Howard G, et al. Factors influencing the decline in stroke mortality: a statement from the American Heart Association/American Stroke Association. Stroke. 2014;45(1):315-53. doi: 10.1161/01.str.0000437068.30550.cf.

3. Titianova E, Velcheva I, Andonova S. Stroke in Bulgaria: Recent problems. Neurosonol Cereb Hemodyn. 2015;11:7-13.

4. Wu CY, Chuang LL, Lin KC, Lee SD, Hong WH. Responsiveness, minimal detectable change, and minimal clinically important difference of the Nottingham Extended Activities of Daily Living Scale in patients with improved performance after stroke rehabilitation. Arch Phys Med Rehabil. 2011;92(8):1281-7. doi: 10.1016/j.apmr.2011.03.008.

5. de Weerd L, Rutgers WA, Groenier KH, van der Meer K. Perceived wellbeing of patients one year post stroke in general practice--recommendations for quality aftercare. BMC Neurol. 2011;11:42. doi: 10.1186/1471-2377-11-42.

6. Tsalta-Mladenov M., Georgieva D., Andonova S. Measuring quality of life in stroke survivors. Russ Neurol J. 2020;25(3):11-6.

7. Lin KC, Fu T, Wu CY, Hsieh CJ. Assessing the stroke-specific quality of life for outcome measurement in stroke rehabilitation: Minimal detectable change and clinically important difference. Health Qual Life Outcomes. 2011;9:5. doi: 10.1186/1477-7525-9-5.

8. Sacco RL, Kasner SE, Broderick JP, Caplan LR, Connors JJ, Culebras A, et al. An updated definition of stroke for the 21st century: a statement for healthcare professionals from the American Heart Association/American Stroke Asso- ciation. Stroke. 2013;44(7):2064-89. doi: 10.1161/ STR.0b013e318296aeca.

9. Brott T, Adams HP Jr., Olinger CP, Marler JR, Barsan WG, Biller J, et al. Measurements of acute cerebral infarction: a clinical examination scale. Stroke. 1989;20(7):864-70. doi: 10.1161/01.str.20.7.864.

10. Broderick JP, Adeoye O, Elm J. Evolution of the modified Rankin scale and its use in future stroke trials. Stroke. 2017;48(7):2007-12. doi: 10.1161/ STROKEAHA.117.017866.

11. Duncan P, Bode R, Lai S, Perera S. Rasch analysis of a new stroke-specific outcome scale: the Stroke Impact Scale. Arch Phys Med Rehabil. 2003;84(7):95063. doi: 10.1016/s0003-9993(03)00035-2.

12. Lai SM, Studenski S, Duncan PW, Perera S. Persisting consequences of stroke measured by the Stroke Impact Scale. Stroke. 2002;33(7):1840-4. doi: 10.1161/01.str.0000019289.15440.f2.

13. Fróes K, Moreno-Valdés M, Lopes D, Silva C. Factors associated with health-related quality of life for adults with stroke sequelae. Arq Neuropsiquiatr. 2011;69(2B):371-6. doi: 10.1590/ s0004-282x2011000300020.

14. Mahran SA, Abdulrahman MA, Janbi FS, Jamalellail RA. The health-related quality of life in stroke survivors: clinical, functional, and psychosocial correlate. Egypt Rheumatol Rehabil. 2015;42(4):188. doi: 10.4103/1110-161X.168198.

15. Dobkin $\mathrm{BH}$. Clinical practice. Rehabilitation after stroke. N Engl J Med. 2005;352(16):1677-84. doi: 10.1056/NEJMcp043511.

16. Zhao LR, Willing A. Enhancing endogenous capacity to repair a stroke-damaged brain: An evolving field for stroke research. Prog Neurobiol. 2018;163164:5-26. doi: 10.1016/j.pneurobio.2018.01.004.

17. 17. Ramos-Lima MJM, Brasileiro IdC, Lima TL, Braga-Neto P. Quality of life after stroke: impact of clinical and sociodemographic factors. Clinics (Sao Paulo). 2018;73:e418-e. doi: 10.6061/clinics/2017/ e418.

18. Portney LG, Watkins MP. Foundations of clinical research: applications to practice. Upper Saddle River, NJ: Pearson/Prentice Hall; 2009.

19. van der Lee JH, Wagenaar RC, Lankhorst GJ, Vogelaar TW, Devillé WL, Bouter LM. Forced use of the upper extremity in chronic stroke patients: results from a single-blind randomized clinical trial. Stroke. 1999;30(11):2369-75. doi: 10.1161/01. str.30.11.2369. 
20. Aprile I, Di Stasio E, Romitelli F, Lancellotti S, Caliandro P, Tonali P, et al. Effects of rehabilitation on quality of life in patients with chronic stroke. Brain Inj. 2008;22(6):451-6. doi: 10.1080/02699050802060639.

21. Ayerbe L, Ayis S, Wolfe CD, Rudd AG. Natural history, predictors and outcomes of depression after stroke: systematic review and meta-analysis. Br J Psychiatry. 2013;202(1):14-21. doi: 10.1192/bjp. bp.111.107664.
22. Al-Qazzaz NK, Ali SH, Ahmad SA, Islam S, Mohamad K. Cognitive impairment and memory dysfunction after a stroke diagnosis: a post-stroke memory assessment. Neuropsychiatr Dis Treat. 2014;10:1677-91. doi: 10.2147/NDT.S67184. 